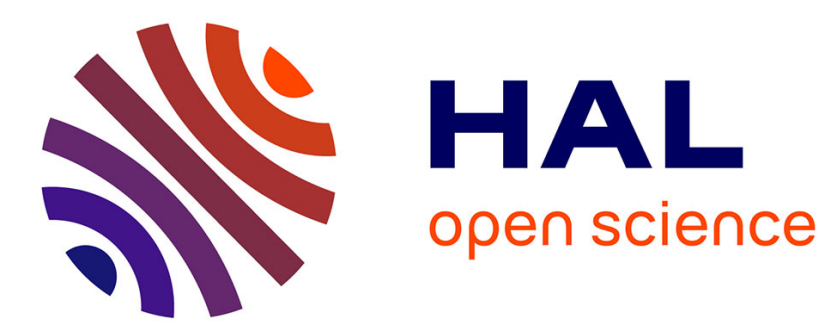

\title{
Preliminary results of the feasibility of hydrogen detection by the use of uncoated silicon microcantilever-based sensors
}

Mohand-Tayeb Boudjiet, Vincent Cuisset, Claude Pellet, Johan Bertrand, Isabelle Dufour

\section{To cite this version:}

Mohand-Tayeb Boudjiet, Vincent Cuisset, Claude Pellet, Johan Bertrand, Isabelle Dufour. Preliminary results of the feasibility of hydrogen detection by the use of uncoated silicon microcantilever-based sensors. International Journal of Hydrogen Energy, 2014, 39, pp.20497-20502. 10.1016/j.ijhydene.2014.03.228 . hal-00967495

\section{HAL Id: hal-00967495 \\ https://hal.science/hal-00967495}

Submitted on 15 Nov 2014

HAL is a multi-disciplinary open access archive for the deposit and dissemination of scientific research documents, whether they are published or not. The documents may come from teaching and research institutions in France or abroad, or from public or private research centers.
L'archive ouverte pluridisciplinaire HAL, est destinée au dépôt et à la diffusion de documents scientifiques de niveau recherche, publiés ou non, émanant des établissements d'enseignement et de recherche français ou étrangers, des laboratoires publics ou privés. 


\title{
PRELIMINARY RESULTS OF THE FEASIBILITY OF HYDROGEN DETECTION BY THE USE OF UNCOATED SILICON MICROCANTILEVER-BASED SENSORS
}

\author{
Boudjiet, M.T. ${ }^{1}$, Cuisset, V. ${ }^{1}$, Pellet, C. ${ }^{1}$, Bertrand, J. ${ }^{2}$, Dufour, I. $^{1}$ \\ ${ }^{1}$ Univ. Bordeaux, IMS, UMR 5218, Talence 33400, France, isabelle.dufour@ims-bordeaux.fr \\ 2 Andra, Châtenay-Malabry 92298, France, johan.bertrand@andra.fr
}

\begin{abstract}
Hydrogen is a key parameter to monitor radioactive disposal facility such as the envisioned French geological repository for nuclear wastes. The use of microcantilevers as chemical sensors usually involves a sensitive layer whose purpose is to selectively sorb the analyte of interest. The sorbed substance can then be detected by monitoring either the resonant frequency shift (dynamic mode) or the quasi-static deflection (static mode). The objective of this paper is to demonstrate the feasibility of eliminating the need for the sensitive layer in the dynamic mode, thereby increasing the long-term reliability. The microcantilever resonant frequency allows probing the mechanical properties (mass density and viscosity) of the surrounding fluid and, thus, to determine the concentration of a species in a binary gaseous. Promising preliminary work has allowed detecting concentration of 200ppm of hydrogen in air with non-optimized geometry of silicon microcantilever with integrated actuation and read-out.
\end{abstract}

\section{INTRODUCTION}

\subsection{Hydrogen release in radioactive waste disposal facility}

Many countries have already selected deep geological disposal as the reference solution for the management of nuclear high level and intermediate level long-lived (HL and IL-LL) radioactive waste. After having concluded a feasibility study on deep geological disposal for HL and IL-LL radioactive waste in 2005, the french National Radioactive Waste Management Agency (Andra) was charged by the Planning Act $\mathrm{n}^{\circ}$ 2006-739 to study the design and the creation of an industrial center for geological disposal called Cigéo which must be reversible for at least a century-long period. Within the framework of this geological repository project, the observation and surveillance must contribute to acquire the knowledge required to run the disposal and its reversible management.

Hydrogen release is expected in the radioactive waste disposal facility. It originates from (i) radioactive waste release (ii) and anoxic corrosion of metallic materials. In fact, some radioactive wastes (containing $\alpha, \beta, \gamma$ radioactivity) resulting from the reprocessing of irradiated fuels are embedded in bitumen matrix. The self-irradiation of the bituminized waste leads mainly to the production of radiolytic hydrogen (75-95 vol. \% of produced radiolytic gas). In the deep geological disposal environments steel components would corrode to more oxidized corrosion products and hydrogen gas. The kinetic of anoxic corrosion of Fe components is not well known, it is actually considered an average rate about $10 \mu \mathrm{m} /$ year. This assumption still awaits experimental verification in the Underground Research Laboratory (sited at Bure in the Meuse district) which aims at studying the feasibility of the reversible geological disposal of high-level and long-lived intermediate-level radioactive waste in the Callovo-Oxfordian clay formation (east of France). Despite the fact that hydrogen releases are expected to be small (in the order of $430 \mathrm{mmol} /$ hour for each intermediate level nuclear waste), when ventilation stops with cell closure, concentrations would slowly and regularly increase. The first calculation gives $4 \%$ hydrogen content in the atmosphere of the radioactive waste cell in less than one year by the end of the oxic corrosion period. 
Monitoring of repository structures contributes to security, safety and reversible management of the repository [1]. It is important as it relates to the guidance of the disposal process and to the corresponding decision-making process. In the upstream repository-design phases, the hydrogen monitoring system was planned to work under normal operating conditions and to withstand radiation exposure in case of an accidental event. There exist different kinds of hydrogen sensors, including those that are commercially available and others still under development [2]. In the present paper, the preliminary results concerning the development of microcantilever-based sensors without sensitive coating for hydrogen detection are presented.

\subsection{Microcantilever-based sensors without sensitive coating}

In recent years, interest in microcantilever-based chemical sensing systems has risen due to their projected high sensitivity [3-7]. The large ratio of surface area to volume makes the microcantilever extremely sensitive to surface processes. For chemical detection, the microcantilever is usually coated with a chemically sensitive layer whose purpose is to selectively sorb the analyte or molecule of interest. The sorbed substance can then be detected by monitoring either the resonant frequency shift (dynamic mode) or the quasi-static deflection (static mode). A simplified way to explain the basic principle of such sensors is to say that in the case of dynamic mode operation, the change in mass associated with the sorption of analyte onto/into the sensitive coating causes a shift in resonant frequency, which may be correlated to the ambient concentration of the target substance. For staticmode operation, the sorption of analyte causes a cantilever deflection that is induced by surface stress or by the tendency of the sensitive coating to expand or contract upon analyte sorption or desorption (modification of strain and stress in the coating). Typical examples of such microsensors for hydrogen detection are: (i) palladium-coated cantilevers operating in static mode [8] and exploiting the expansion of palladium associated to the formation of hydride compounds during hydrogenation, (ii) and resonator based-microcantilever operating in dynamic mode, using carbone nanotubes (CNTs) as sensitive layer [9] and exploiting hydrogen storage capacity of this material.

The major drawbacks of the use of a sensitive coating come from the fact that they are subjected to environmental effects including temperature, humidity and aging effects. Then, the limited long-term stability of the coatings and the resulting aging affect the reliability of the sensor. To eliminate problems associated with the sensitive coatings, an alternative method to detect and quantify chemical species without the use of a sensitive coating has been proposed [10]. In this paper this principle will be tested for hydrogen detection.

\section{MODELING}

The geometry of the cantilever (Fig.1) is defined by the width $b$, thickness $h$, and length $L$. Coordinate $x$ is measured along the beam length. The properties of the cantilever material are the Young's modulus $E$ and the mass density $\rho$. The fluid (gas or liquid) properties are defined as $\rho_{f}$, the fluid's mass density, and $\eta$, the fluid's dynamic viscosity.

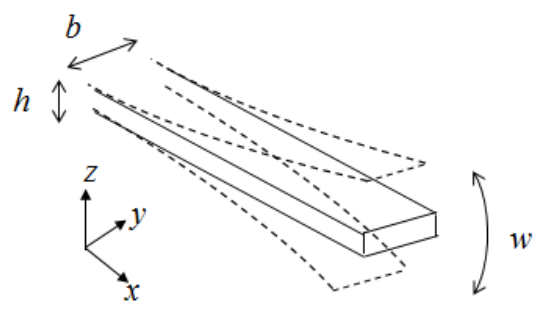

Figure 1. Schematic representation of the microcantilever geometry and of the transverse bending vibration 


\subsection{Resonant frequency shift due to modification of fluid properties}

When a microcantilever vibrates in a viscous fluid (gas or liquid), the fluid offers resistance to the motion. The force per unit length, $F_{\text {fluid, }}$, which is the consequence of all normal and tangential stresses (hydrodynamic pressure and viscous shear) exerted by the fluid on all the surfaces of the cantilever, can be written in the frequency domain as [11]:

$$
F_{\text {fluid }}(x, \omega)=-\left[j \omega g_{1}(x, \omega)-\omega^{2} g_{2}(x, \omega)\right] w(x, \omega)
$$

where $x$ is the longitudinal coordinate, $\omega$ the radial frequency of vibration, $w$ the microcantilever deflection, $j$ is the unit imaginary number, and $g_{1}$ and $g_{2}$ are functions depending on the fluid properties and the microcantilever cross-sectional geometry. They may be interpreted, respectively, as the distributed damping coefficient of the fluid and the distributed effective fluid mass (per unit length of beam). In general, these quantities are both frequency-dependent and $x$-dependent; however, in what follows $g_{1}$ and $g_{2}$ will be assumed uniform in $x$ as this assumption has been shown to be sufficiently accurate in many cases of practical interest [12].

It is common to normalize $F_{\text {fluid }}$ into a dimensionless form called the hydrodynamic function, $\Gamma(\omega)=\Gamma_{\mathrm{r}}(\omega)-j \Gamma_{\mathrm{i}}(\omega)$, where [13]:

$$
\begin{aligned}
& \Gamma_{r}(\omega)=\frac{4}{\pi \rho_{f} b^{2}} g_{2}(\omega) \\
& \Gamma_{i}(\omega)=\frac{4}{\pi \rho_{f} b^{2}} \frac{g_{1}(\omega)}{\omega}
\end{aligned}
$$

The hydrodynamic function, $\Gamma$, represents the total hydrodynamic force per unit length normalized by the force per unit length required to excite (at the same amplitude and frequency) a circular cylindrical volume of diameter $b$ and density $\rho_{f}$. The subscripts $r$ and $i$ in Eqs. 2-3 denote the real and imaginary parts of the hydrodynamic function, respectively.

The fluid effects (viscous term $g_{1}$, and inertial term $g_{2}$ ) influence the dynamic response of the beam; in particular, the resonant frequency, $f_{r}$, and the quality factor associated with viscous losses, $Q_{v i s c}$, may be expressed as [12-13]:

$$
\begin{gathered}
f_{r}=f_{0, \text { vac }} \frac{1}{\sqrt{1+L g_{2} / m}} \sqrt{1-\frac{1}{2 Q^{2}}} \\
Q_{\text {visc }}=\frac{2 \pi \sqrt{1+L g_{2} / m}}{L g_{1} / m} f_{0, \text { vac }}
\end{gathered}
$$

where $f_{0, v a c}$ is the undamped natural frequency of the microcantilever in vacuum, $f_{0, \text { vac }}=\left(1.875^{2} h /\left(2 \pi L^{2}\right)\right) \sqrt{E /(12 \rho)}, Q$ is the total quality factor of the cantilever/fluid system (incorporating all losses), $m$ is the total microcantilever mass and $L$ is the microcantilever length.

The hydrodynamic function of a transversely vibrating beam of ribbon-like cross-section (i.e., width $b$ much larger than the thickness $h$ ) was previously determined in [14] by using the method of moments to solve for the velocity field in the fluid and in [13] by performing a fit of the numerical results of [14]. 
A more accurate approximation of the total hydrodynamic force acting on a transversely vibrating microcantilever in liquids should include both the effects of the pressure and shear stress exerted by the fluid on all faces of the beam of finite cross-sectional dimensions. Due to the symmetry of the problem, the hydrodynamic forces acting in the $x$ or $y$ direction on a beam with a rectangular crosssection vibrating transversely will cancel each other out. Only the hydrodynamic forces acting in the $z$ direction will then affect the response of the beam. These forces are the pressure forces acting on the large faces (of dimension $b$ ) and the shear forces acting on the small faces (of dimension $h$ ). It can be assumed that the microcantilever is long enough so that the hydrodynamic force acting on the surface at the unsupported end of the beam is negligible. Recently, results for the hydrodynamic function in the case of transverse bending that accounted for both the beam's aspect ratio $(h / b)$ and the Reynolds number $(\mathrm{Re})$ of the fluid flow have been published [15]. A Taylor series of the analytical function or a fitting of the numerical results can be made, and then the hydrodynamic function $\Gamma$ can be written as

$$
\begin{aligned}
& \Gamma_{r}=a_{0}(b / h)+a_{1}(b / h) \frac{1}{\sqrt{\operatorname{Re}}}+a_{2}(b / h) \frac{1}{\operatorname{Re}} \\
& \Gamma_{i}=b_{0}(b / h)+b_{1}(b / h) \frac{1}{\sqrt{\operatorname{Re}}}+b_{2}(b / h) \frac{1}{\operatorname{Re}}
\end{aligned}
$$

with $a_{0}, a_{1}, a_{2}, b_{0}, b_{1}$ and $b_{2}$ being functions of aspect ratio $b / h$, and the Reynolds number, Re, defined as:

$$
\operatorname{Re}=\frac{\omega b^{2} \rho_{f}}{4 \eta}
$$

For the ribbon-like case, $a_{0}, a_{1}, a_{2}, b_{0}, b_{1}$ and $b_{2}$ have constant numerical values. In [16] a fit of the results of [13] has been made and the obtained numerical values are:

$$
\begin{array}{lll}
a_{0}=1.0553 & a_{1}=3.7997 & a_{2}=0 \\
b_{0}=0 & b_{1}=3.8018 & b_{2}=2.7364
\end{array}
$$

The above equations illustrate the fact that the resonant frequency depends on the fluid properties via the Reynolds number, Re, which appears in the hydrodynamic function $\Gamma$ (Eqs. 6-7) and consequently in the terms $g_{1}$ and $g_{2}$.

\subsection{Fluid properties of binary mixture}

First, in order to apply the theoretical equations, it is necessary to know the mass density, $\rho_{f}$, and viscosity, $\eta$, of a binary mixture.

Using the definition of the mass density, in the case of a binary mixture, it can be expressed as a function of the mass densities of each component:

$$
\rho_{f}=x_{1} \rho_{1}+\left(1-x_{1}\right) \rho_{2}
$$

with $\rho_{1}$ and $\rho_{2}$ the mass densities of gas 1 and gas 2 , respectively, and $x_{1}$ the volume fraction of gas 1 .

Contrary to the case of mass density, the viscosity of a binary mixture is not easy to obtain using the viscosity definition. An approximate expression for the viscosity of gas mixtures was derived by Sutherland in 1895. It is based on a simple mean-free-path model and, for a binary gas mixture, is given by [17]: 


$$
\eta=\frac{x_{1} \eta_{1}}{x_{1}+\left(1-x_{1}\right) \Phi_{12}}+\frac{\left(1-x_{1}\right) \eta_{2}}{1-x_{1}+x_{1} \Phi_{21}}
$$

with $\eta_{1}$ and $\eta_{2}$ being the viscosities of gas 1 and gas 2 , respectively.

Concerning the expressions for $\Phi_{12}$ and $\Phi_{21}$, many attempts to predict these parameters have been made. Wilke derived the following expressions [18]:

$$
\begin{aligned}
& \Phi_{12}=\left[8\left(1+\frac{M_{1}}{M_{2}}\right)\right]^{-1 / 2}\left[1+\left(\frac{\eta_{1}}{\eta_{2}}\right)^{1 / 2}\left(\frac{M_{2}}{M_{1}}\right)^{1 / 4}\right]^{2} \\
& \Phi_{21}=\left[8\left(1+\frac{M_{2}}{M_{1}}\right)\right]^{-1 / 2}\left[1+\left(\frac{\eta_{2}}{\eta_{1}}\right)^{1 / 2}\left(\frac{M_{1}}{M_{2}}\right)^{1 / 4}\right]^{2}
\end{aligned}
$$

where $M_{1}$ and $M_{2}$ are the molar masses of the gases.

The average deviation between calculated and experimental data for thirteen mixtures involving different gases $\left(\mathrm{CO}_{2}, \mathrm{O}_{2}, \mathrm{CO}, \mathrm{H}_{2}, \mathrm{CH}_{4}, \mathrm{~N}_{2}\right.$, etc.) is $1.9 \%$ [18]. Brokaw gave other more complicated expressions resulting in almost the same numerical results [19]: for 280 mixtures involving 25 gas pairs (including $\mathrm{H}_{2} / \mathrm{N}_{2}$ ), the average error when comparing with experimental data is $0.7 \%$ [19]. Both of these methods use only the viscosity and molecular mass of the two pure components.

\subsection{Numerical frequency shift for hydrogen detection}

Considering that hydrogen and nitrogen are perfect gases, their mass densities can be calculated for a temperature of $20^{\circ} \mathrm{C}$ and a pressure of $10^{5} \mathrm{~Pa}$. For these temperature and pressure conditions, the mass density of hydrogen and nitrogen are, respectively:

$$
\rho\left(\mathrm{H}_{2}\right)=0.082055 \mathrm{~kg} / \mathrm{m}^{3} ; \rho\left(\mathrm{N}_{2}\right)=1.1488 \mathrm{~kg} / \mathrm{m}^{3}
$$

The viscosities of the gases also depend on the temperature [20]:

$$
\eta=2.669310^{-6} \frac{\sqrt{M T}}{\sigma^{2} \Omega^{(2,2)^{*}}}
$$

with $M$ the molar mass $\left(\mathrm{g} \cdot \mathrm{mol}^{-1}\right), T$ the temperature $(\mathrm{K}), \sigma$ a length for the expression of the interaction potential $(\AA)$ and $\Omega^{(2,2)^{*}}$ the double normalized collision integral for the Lennard-Jones potential:

$$
\Omega^{(2,2)^{*}}=1.1614\left(T^{*}\right)^{-0.1487}+0.5249 e^{-0.7732 T^{*}}+2.1618 e^{-2.4378 T^{*}}
$$

with the normalized temperature $T^{*}$ defined by:

$$
T^{*}=\frac{k T}{\varepsilon}
$$

where $k$ is the Boltzmann constant and $\varepsilon$ the energy in the action potential.

Using equations 15-17 and the numerical values of the different parameters for each gas, the viscosities at $20^{\circ} \mathrm{C}$ for hydrogen and nitrogen are, respectively: 
$\eta_{\mathrm{H}_{2}}=8.7106 \mu$ Pa.s $; \eta_{\mathrm{N}_{2}}=17.555 \mu$ Pa.s

Using equations 10 , it is possible to calculate the mass density of the binary mixture of $1 \%$ of hydrogen in nitrogen and its relative variation compared to pure nitrogen:

$\rho_{f}\left(1 \% \mathrm{H}_{2} / 99 \% \mathrm{~N}_{2}\right)=1.1381 \mathrm{~kg} / \mathrm{m}^{3} ; \frac{d \rho_{f}\left(1 \% \mathrm{H}_{2} / 99 \% \mathrm{~N}_{2}\right)}{\rho_{f}\left(100 \% \mathrm{~N}_{2}\right)}=-0.928 \%$

Using equation 11 , it is possible to calculate the viscosity of the binary mixture of $1 \%$ of hydrogen in nitrogen and its relative variation compared to pure nitrogen:

$$
\eta\left(1 \% \mathrm{H}_{2} / 99 \% \mathrm{~N}_{2}\right)=17.548 \mu \mathrm{Pa} . \mathrm{s} ; \frac{d \eta\left(1 \% \mathrm{H}_{2} / 99 \% \mathrm{~N}_{2}\right)}{\eta\left(100 \% \mathrm{~N}_{2}\right)}=-0.015 \%
$$

Considering small variations of fluid density and fluid viscosity, it is possible to obtain the analytical expression of the resonant frequency shift due to these fluid property variations. Using equations 4-9, the resonant frequency shift can be approximated with a first-order approximation:

$$
\frac{d f_{r}}{f_{r}}=-\frac{\pi}{8} \frac{\rho_{f}}{\rho} \frac{b}{h}\left(a_{0} \frac{d \rho_{f}}{\rho_{f}}+\frac{a_{1}}{2 \sqrt{2 \operatorname{Re}}} \frac{d \eta}{\eta}\right)
$$

Using this equation, the numerical results obtained in equations 19-20 and the fact that the Reynolds number of microcantilever vibrating in gas is usually high, it comes that the resonant frequency shift is essentially due to the fluid mass density variation. Moreover, from equation 21 , one may conclude that the relative frequency shift due to the mass density variation of the gas increases with the ratio of the microcantilever width to the microcantilever thickness $(b / h)$.

To estimate the magnitude of the relative resonant frequency shift due to the increase of $1 \%$ of hydrogen in nitrogen, let's consider a silicon cantilever $\left(\rho=2330 \mathrm{~kg} / \mathrm{m}^{3}\right)$ with a ratio of width to thickness $(b / h)$ equal to 25 . Using this particular case, a relative resonant frequency shift of $50 \mathrm{ppm}$ is obtained. This variation is small but such measurement is proven to be possible. Then test of hydrogen detection has been conducted.

\section{EXPERIMENTS}

Examples for the detection of hydrogen in nitrogen using silicon cantilevers not dedicated to this application have been made in order to validate the principle of hydrogen detection with uncoated microcantilever.

\subsection{Microcantilever design and gas line setup}

The microcantilevers have been fabricated by ESIEE group (Paris, France, www.esiee.fr) using silicon technologies [21].

The excitation of the microcantilever is made by electromagnetic actuation. The excitation arises due to the interaction between a magnetic field created by a magnet placed close to the chip and an electrical current passing through a conducting strip placed on the microcantilever. The Lorentz electromagnetic force induces the structural motion. In order to detect the microcantilever oscillation, semiconductor strain gauges (boron-doped piezoresistors) are fabricated during the process. They are arranged in a half Wheatstone bridge configuration: a first gauge is located where the strains are maximum (the clamped-end of the beam) and the other is on the rigid substrate (Fig. 2). 


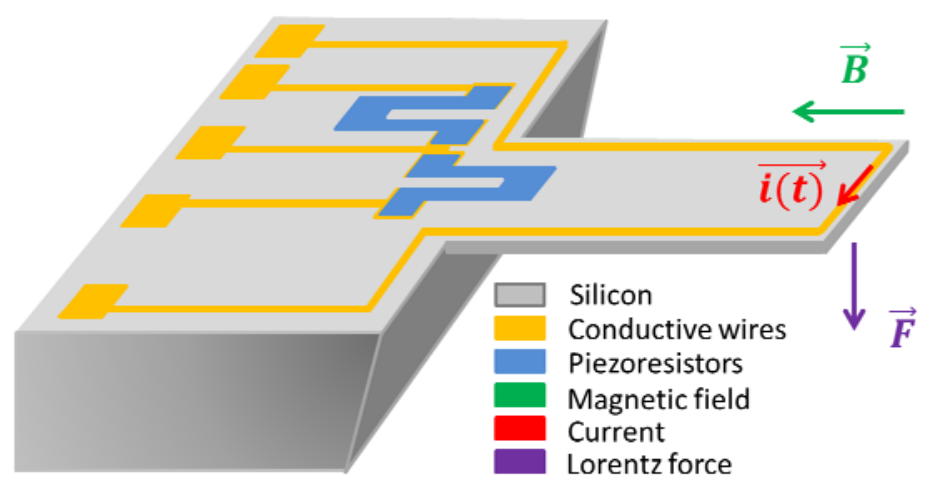

Figure 2. Schematic representation of the microcantilever electromagnetic actuation and piezoresistive read-out

The way to measure the resonant frequency shift is to actuate the microcantilever at a fixed frequency close to the resonant frequency, and to read out the piezoresistive signal phase. When the resonant frequency shifts, the phase of the microcantilever signal is modified. Near the resonant frequency, the phase is almost a linear function of the frequency; thus, the measurement of the phase shift at a fixed frequency allows one to determine the resonant frequency shift.

The microcantilevers are placed in a gas chamber (total volume: $500 \mu \mathrm{l}$ ) under a controlled gas flow $\left(100 \mathrm{~cm}^{3} / \mathrm{min}\right)$. Gas streams containing binary mixtures of the desired species (hydrogen) and nitrogen are fed to the chamber using bottles of gas and a set of mass-flow controllers.

\subsection{Hydrogen detection}

The silicon cantilever used for this test has a length of $2 \mathrm{~mm}$, a width of $400 \mu \mathrm{m}$ and a thickness of $15 \mu \mathrm{m}$. The resonance frequency of the uncoated sensor is approximately $5 \mathrm{kHz}$.

Figure 3 shows examples of resonant frequency shift measurements for various hydrogen concentrations in nitrogen $(2 \%, 1 \%, 0.6 \%$ and $0.2 \%)$.

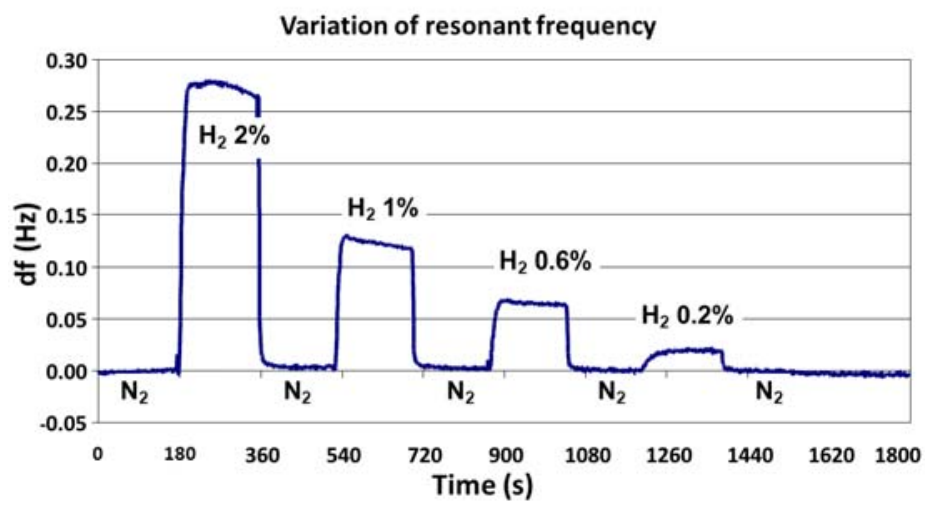

Figure 3. Example of detection of hydrogen in nitrogen with an uncoated $2000 \times 400 \times 15 \mu \mathrm{m}^{3}$ silicon cantilever

As predicted by Eq. 21, due to the fact that hydrogen is lighter than nitrogen, the resonant frequency increases with the hydrogen concentration. The resonant frequency shift is less than the expected one. This is probably due to the fact that some gas adsorption occurs on the surface of the cantilever. 
Nevertheless, other measurements made with different cantilever geometries have proven that, as stated by the theoretical equations, the relative resonant frequency shift is almost proportional to the ratio (width/thickness) and to the variation of the mass density of the hydrogen/nitrogen mixture [22].

In Figure 3, the duration of each concentration step is 3 minutes, this proves that the response time of the sensor without sensitive coating is very small. The $0-90 \%$ sensor response time to $1 \%$ of $_{2} \mathrm{H}_{2} \mathrm{~N}_{2}$ is $12 \mathrm{~s}$ which is much faster than measurements made with the same gas chamber with polymer coated cantilevers for VOC detection (more than $1 \mathrm{~min}$ ) [23] and palladium coated cantilever in static mode for hydrogen detection (90 s) [8].

In order to verify that the measurement is due to a modification of the mass density of the gas, similar test have been conducted with carbon dioxide in nitrogen. The results corresponding to different concentrations $(2 \%, 1.5 \%, 1 \%$ and $0.5 \%)$ are presented in Figure 4. In this case, the resonant frequency decreases with carbon dioxide concentration, due to the fact that this gas is heavier than nitrogen. This experiment allows validating that the measured shifts of resonant frequency are induced by the modification of the mass density of the gas.

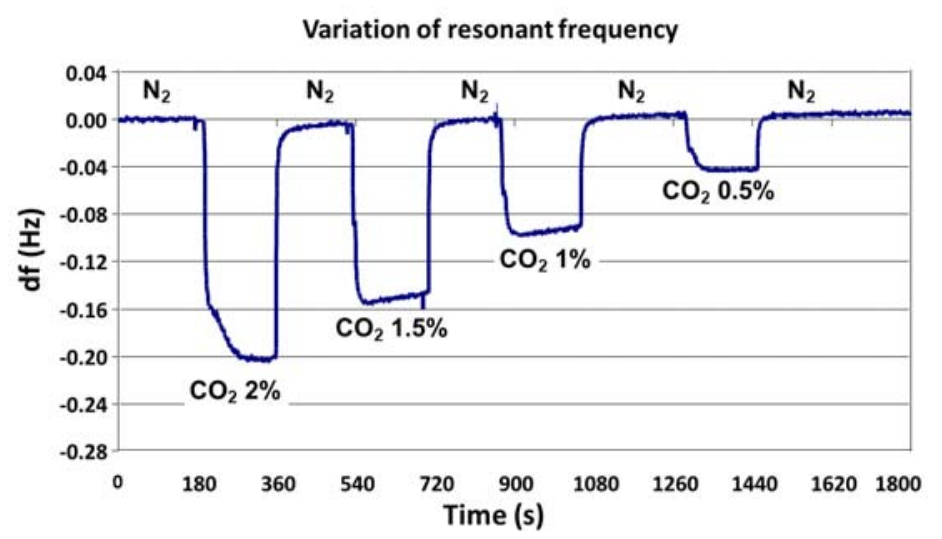

Figure 4. Example of detection of carbon dioxide in nitrogen with an uncoated $2000 \times 400 \times 15 \mu \mathrm{m}^{3}$ silicon cantilever

The sensor noise has been quantified by calculating the standard deviation of measurement on one concentration step: it is equal to $0.9 \mathrm{mHz}$. The estimated limit of detection or the resolution corresponds to a frequency shift three times larger than the noise, which corresponds to $0.02 \% \mathrm{of}_{2}$ in $\mathrm{N}_{2}(200 \mathrm{ppm})$, since a resonant frequency shift of $20 \mathrm{mHz}$ has been measured for $0.2 \%$ of $\mathrm{H}_{2}$ in $\mathrm{N}_{2}$. In order to validate experimentally this estimated value of the limit of detection with this cantilever, measurements with smaller hydrogen concentrations have been conducted (Fig. 5). It can be seen that, without any signal processing, the detection of $200 \mathrm{ppm}$ of hydrogen is possible and probably the detection of smaller concentration can be achieved.

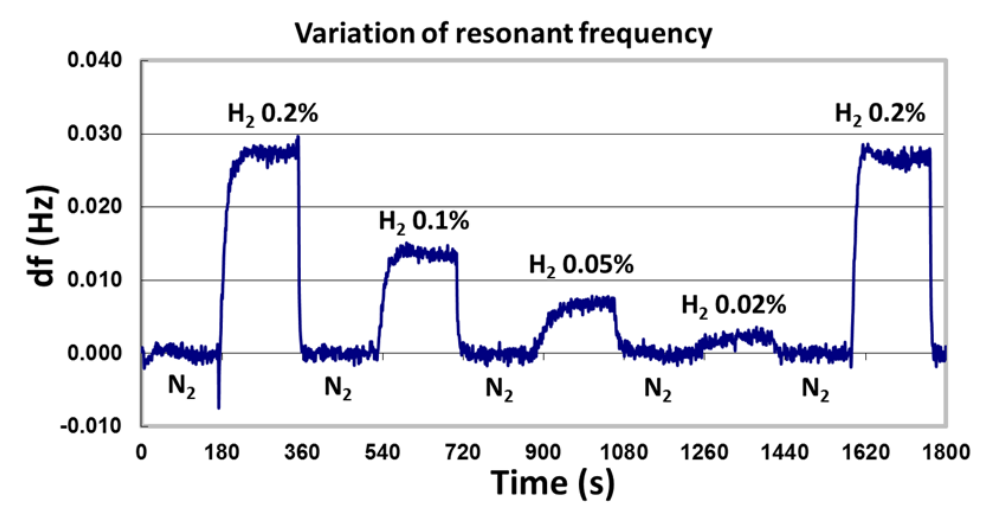


Figure 5. Example of detection of small concentration of hydrogen in nitrogen with an uncoated $2000 \times 400 \times 15 \mu \mathrm{m}^{3}$ silicon cantilever

The experimental conditions of the measurement presented in Figure 5 were quite different from the one of Figure 3: the gas flow has been increased from $100 \mathrm{~mL} / \mathrm{min}$ to $250 \mathrm{~mL} / \mathrm{min}$ in order to be able to generate the small concentration of $0.02 \%$ of $\mathrm{H}_{2}$, and the temperature was approximately $26^{\circ} \mathrm{C}$ instead of $23^{\circ} \mathrm{C}$. These experimental conditions can explain the difference of the resonant frequency shift corresponding to $0.2 \%$ of $\mathrm{H}_{2}(0.020 \mathrm{~Hz}$ and $0.028 \mathrm{~Hz}$ in Figure 3 and Figure 5, respectively). The sensitivity of such sensors depends on the temperature because both the gas density and the cantilever Young's modulus are temperature dependent. Then, in order to have repeatability in the measurement the temperature has to be the same (this was the case for the two resonant frequency shifts corresponding to $0.2 \%$ of $\mathrm{H}_{2}$ in Figure 5).

\section{CONCLUSION AND FUTURE WORKS}

Compared to classical resonant microcantilever-based chemical sensors with sensitive coatings, the uncoated microsensors exhibit shorter response times because there is no analyte sorption into the coating affecting the sensor response. Moreover, the absence of the sensitive coating leads to a more reliable and reversible behavior because there is no significant absorption and desorption phenomena. The above results indicate that uncoated microsensors may serve as viable devices for the detection of specific concentrations of one gas in a binary mixture. The sensitivity and resolution of such sensors will be larger for those cases in which the difference between the mass densities of the two gases is higher (which is the case for hydrogen in nitrogen or air). The major drawbacks of such sensors is that there is no intrinsic selectivity; thus, these sensors can only be used for specific applications, such as when monitoring environments in which it is known that only one gas concentration can vary, while the relative concentrations of the other gases remain fixed. Another intrinsic drawback concerns the sensitivity which is smaller than coated microcantilevers, although, as presented in this paper, the sensitivity is sufficient to detect 200ppm of hydrogen in nitrogen.

These preliminary results are promising and the future work will consist to optimize the geometry of the vibrating microstructure (increase of the width to thickness ratio, paddle-shaped geometry, etc.) in order to increase the sensitivity, decrease the noise and then improve the limit of detection. Another study will concern the influence of parasitic variations (temperature, third gas, etc.) and the compensation of these variations.

\section{REFERENCE}

1. Buschaert, S., Lesoille-Delepine, S., Bertrand, J., Mayer, S. and Landais P., Developing the tools for geologic repository monitoring - Andra's monitoring R\&D program, Proc. of the WM2012 conference, Phoenix, 2012.

2. Hübert, T., Boon-Brett, L., Black, G., Banach, U., Hydrogen Sensors- A review, Sensors and Actuators B, 157, 2011, pp. 329-352.

3. Goeders, K.M., Colton, J.S. and Bottomley, L. A., Microcantilevers: sensing chemical interactions via mechanical motion, Chemical Reviews, 108, 2008, pp. 522-542 .

4. Boisen, A., Dohn, S., Keller, S.S., Schmid, S. and Tenje, M., Cantilever-like micromechanical sensors, Reports on Progress in Physics, 74, 2011, pp. 1-31.

5. Li, X. and Lee, D.W., Integrated microcantilevers for high-resolution sensing and probing, Measurement Science and Technology, 23, 2012, 022001.

6. Sepaniak, M., Datskos, P., Lavrik, N. and Tipple, C., Microcantilever transducers: a new approach in sensor technology, Analytical Chemistry, 74, 2002, pp. 568-575. 
7. Zhu, Q., Microcantilever sensors in biological and chemical detections, Sensors \& Transducers Journal, 125, No. 2, 2011, pp. 1-21.

8. Baselt, D.R., Fruhberger, B., Klaassen, E., Cemalovic, S., Britton Jr., C.L., Patel. S.V., Mlsna, T.E., McCorkle, D. and Warmack. B., Design and performance of a microcantilever-based hydrogen sensor, Sensors and Actuators B, 88, 2003, pp. 120-131.

9. Ono, T., Li, X., Miyashita, H. and Esashi, M., Mass sensing of adsorbed molecules in subpicogram sample with ultrathin silicon resonator, Review of Scientific Instruments, 74, 2003, pp. $1240-1243$.

10. Tétin, S., Caillard, B., Ménil, F., Debéda, H., Lucat, C., Pellet, C. and Dufour, I., Modeling and performance of uncoated microcantilever-based chemical sensors, Sensors and Actuators B, 143, 2010, pp. 555-560.

11. Blevins, R.D., Formulas for natural frequency and mode shape, Krieger Publishing Company, Reprint edition 2001.

12. Chon, J.W.M., Mulvaney, P. and Sader, J.E., Experimental validation of theoretical models for the frequency response of atomic force microscope cantilever beams immersed in fluids, Journal of Applied Physics, 87, 2000, pp. 3978-3988.

13. Sader, J., Frequency response of cantilever beams immersed in viscous fluids with applications to the atomic force microscope, Journal of Applied Physics, 84, 1998, pp. 64-76.

14. Tuck, E., Calculation of unsteady flows due to small motions of cylinders in a viscous fluid, Journal of Engineering Mathematics, 3, No. 1, 1969, pp. 29-44.

15. Brumley, D., Willcox, M. and Sader, J., Oscillation of cylinders of rectangular cross section immersed in fluid, Physics of Fluids, 22, 2010, 052001.

16. Maali, A., Hurth, C., Boisgard, R., Jai, C., Cohen-Bouhacina, T. and Aimé, J.-P., Hydrodynamics of oscillating atomic force microscopy cantilevers in viscous fluids, Journal of Applied Physics, 97, 2005, 074907.

17. Sutherland, W., The viscosity of mixed gases, Philosophical Magazine and Journal of Science, $\mathbf{4 0 ,}$ 1895, pp. 421-431.

18. Wilke, C.R, A viscosity equation for gas mixtures, Journal of Chemical physics, 18, 1950, pp. 517-518.

19. Brokaw, P.S., Predicting transport properties of dilute gases, Industrial Engineering Chemistry Process Design and Development, 8, 1968, pp. 240-253.

20. Gosse, J., Propriétés de transport des gaz à pression modérée, Techniques de l'ingénieur, traité Constantes physico-chimiques, K 425.

21. Fadel, L., Lochon, F., Dufour, I. and Français O., Chemical sensing: millimeter size resonant microcantilever performance, Journal of Micromechanics and Microengineering, 14, 2004, pp. S23-S30.

22. Cuisset, V., 'Utilisation de micropoutres sans couche sensible en vibration pour la détection d'espèces gazeuses. Rélaisation d'un réseau gaz pilotable', Mémoire de diplôme d'ingénieur CNAM, $20^{\text {th }}$ july 2010.

23. Lochon, F., Fadel, L., Dufour, I., Rebière, D., Pistré, J., Silicon made resonant microcantilever: dependence of the chemical sensing performances on the sensitive coating thickness, Materials Science \& Engineering C, 26, 2006, pp. 348-353. 